'Habit-like' attentional prioritisation of reward-related stimuli

Jenny T. Le, Poppy Watson, \& Mike E. Le Pelley

School of Psychology, UNSW Sydney, Sydney, Australia

Corresponding author:

Jenny T. Le

School of Psychology, UNSW Sydney

Sydney NSW 2052

Australia

Author note: This research was funded by Australian Research Council grants DP170101715 and DP200101314. Poppy Watson was supported by an Australian Research Council

Discovery Early Career Researcher Award (DE200100591). Experiment scripts and raw data are available at https://osf.io/nuaxg.

Word count: 11,817 words 


\begin{abstract}
Stimuli associated with rewards (money, drugs, food) can acquire the ability to capture our attention independently of, and indeed contrary to, our goals and intentions. Here, we examined whether this process of attentional prioritisation is 'habit-like' - namely, whether attentional prioritisation of reward-related cues is insensitive to changes in the value of the reward itself. To this end, we incorporated an instructed value-switch (Experiment 1), devaluation (Experiment 2a), and 'super-valuation' (Experiment 2b) into a visual search task, using eye-tracking to examine attentional prioritisation of stimuli that signal high- and lowvalue rewards. In Experiment 1, following a value-switch manipulation, we found that attentional prioritisation of a high-value stimulus could not be overcome with knowledge of the new values of outcomes alone. Only when provided with further experience of receiving the outcomes could patterns of attentional prioritisation of high- and low-value stimuli switch, in line with the current value of the outcome they signalled. In Experiment $2 \mathrm{a}$ and $2 \mathrm{~b}$, we found that attentional prioritisation of high- and low-value stimuli was insensitive to the devaluation of a previously high-value outcome, and super-valuation of a previously lowvalue outcome, even when participants were provided with further experience of receiving that outcome. Our findings suggest that - under some conditions - attentional prioritisation may fit the definition of a 'habit-like' response. By taking a new approach to studying habits in humans, the current findings provide a framework for future work to further understand attentional influences on behaviour.
\end{abstract}




\section{'Habit-like' attentional prioritisation of reward-related stimuli}

Our attention often operates in a goal-directed way, filtering information from the world and prioritising stimuli that are relevant to our current goals (Yantis, 2000). For example, if we needed to buy salad at the supermarket, our attention might scan ahead for displays of vegetables so that we can locate them faster. Notably, attention can also operate independently of our goals, with recent work demonstrating that prior experiences with rewards can exert an influence on the likelihood that stimuli will automatically capture attention (see reviews by Anderson, 2016; Failing \& Theeuwes, 2018; Le Pelley et al., 2016; Rusz et al., 2020). Imagine that in the past, we have eaten a particular type of chocolate many times, and always found it delicious. On our way to the vegetable section, our attention may be captured by the wrapper of this rewarding chocolate, even though our goal is to look for salad. This fundamental effect of reward on attention is notable because the modern world is filled with reward cues: wrappers on high-calorie foods, logos of restaurant chains, billboards showing attractive models, advertisements for alcohol and cigarettes, and the bright flashing lights of gambling machines. Through repeated pairings with rewards (pleasurable 'highs', feelings of satiation, monetary wins), it has been suggested that these reward cues can become 'motivational magnets' that have the power to elicit approach behaviours (Berridge \& Robinson, 1998).

Many different procedures have been developed to study the influence of reward learning on attentional priority (see reviews by Le Pelley et al., 2016; Rusz et al., 2020). Here we focus on a visual search procedure used by Pearson et al. (2016), which formed the basis of the current study. This procedure uses eye-tracking as a measure of spatial attention, since eye-movements (saccades) to a given location are closely preceded by shifts in attention to that location (Deubel \& Schneider, 1996). On each trial, participants are presented with an array of shapes; one diamond (the target) and several circles. Participants must make a rapid 
saccade to the diamond target to earn a reward. The array also features one coloured distractor circle (either orange or blue; all other shapes are grey), with the colour of the distractor signalling the magnitude of reward available for a correct response (e.g., either 10 or 500 points). Importantly, while the distractor signals the magnitude of reward available on a given trial, participants are informed that the reward will be omitted if they ever look at the coloured distractor. Hence, looking at distractors is counterproductive to participants' goal of earning points in this task. Yet numerous studies have found that this exactly is what participants do (e.g., Le Pelley et al., 2015; Pearson et al., 2015, 2016; Watson et al., 2019a, 2019b, 2020). Notably, participants are more likely to have their attention (and gaze) captured by the distractor that signals a high-value reward, even though this results in the cancellation of a larger reward (relative to looking at the distractor that signals a low-value reward; Pearson et al., 2016). Moreover, the 'attention-grabbing' nature of the distractors (particularly the distractor that signals a high-value reward) persists even when rewards are subsequently removed (Watson et al., 2019b).

These findings suggest that the human attentional system prioritises stimuli that have learned associations with rewards, even when doing so is counterproductive to our goals, and when the rewards themselves are no longer delivered. Moreover, stimuli signalling highvalue rewards compete more effectively for attentional prioritisation than do signals of lowvalue rewards. This modulating influence of reward learning has been termed valuemodulated attentional capture (VMAC; Le Pelley et al., 2015), and has been well-replicated in studies of eye-movements (e.g., Le Pelley et al., 2015; Pearson et al., 2015; Failing et al., 2015), accuracy and latency of manual responses (e.g., Anderson \& Halpern, 2017; Le Pelley et al., 2019; Raymond \& O'Brien, 2009; Watson et al., 2019), and neuroimaging (e.g., Hickey et al., 2010; Qi et al., 2013). 


\section{'Habit-like' attention}

The findings outlined above demonstrate that reward-associated stimuli can capture our attention independently of, and indeed contrary to, our goals and intentions. Viewed another way, these findings suggest that reward-related stimuli can automatically elicit a conditioned attentional prioritisation response. Conceptualising attentional prioritisation as a 'response' that can be conditioned through learned associations with reward raises a question that is central to the current study: what is the nature of the process by which stimuli elicit these conditioned attentional responses? More specifically, we investigate whether prioritisation is mediated by retrieval of the current value of the outcome signalled by a stimulus, or whether instead prioritisation becomes divorced from the value of the events involved; that is, whether attention can become 'habit-like'.

Habits have typically been studied in the field of behavioural neuroscience as distinct from goal-directed behaviours. Goal-directed behaviours are targeted at achieving current goals and obtaining valued outcomes - that is, goal-directed behaviour is mediated by a mental representation of the outcome, and hence is sensitive to the current value of that outcome (Dickinson \& Balleine, 1994). On the other hand, habitual behaviours are elicited 'automatically' as a result of stable and extensive prior experience of receiving reward for making a particular response in the presence of a particular stimulus. That is, habits come to be driven by conditioned stimulus-response ( $\mathrm{S}-\mathrm{R})$ associations. Critically, this means that habitual behaviour is not mediated by a representation of the outcome, so habits are insensitive to changes in the value of that outcome (Dickinson, 1985).

The distinction between goal-directed and habitual behaviours has typically been studied in rodents using the outcome devaluation procedure (Adams \& Dickinson, 1981). In the training phase of this procedure, an instrumental response (e.g., a lever press) is reinforced by the delivery of a reward outcome (such as a food pellet). Following the training 
phase, the outcome is devalued; for example rats might be fed to satiety, thus reducing the value of food pellets. The impact of this devaluation on conditioned lever-pressing is then tested in extinction (i.e., food pellets are no longer delivered). If behaviour is goal-directed (mediated by a representation of the outcome), the rat should reduce lever-pressing following devaluation, in line with the reduced value of the food pellet outcome. By contrast, if reinforcement of lever-pressing during the training phase results in behaviour becoming habitual, then the sight of the lever stimulus during the test phase would automatically elicit a lever-pressing response, even though the food pellet is no longer valuable. That is, the rat's behaviour would be automatically driven by a conditioned S-R association (see lever $\rightarrow$ press lever), independent of the current value of the outcome. Studies using this outcome devaluation procedure show that while behaviours can start out as being outcome-mediated after a small amount of initial training, with stable and extended training behaviours can transition to become outcome-independent habits (Adams, 1982; Dickinson et al., 1995).

Returning to attentional prioritisation of reward-associated stimuli in humans, the VMAC effect shows some features that might be expected of a habit-like response: it increases with extended training (Pearson et al., 2015) and, as noted earlier, seems to result from an automatic and involuntary behavioural process, as it can operate independently of (and indeed, contrary to) an individual's goals. Such findings have led to the description of attentional prioritisation of reward-related stimuli as reflecting an 'attentional habit' (Anderson, 2016; Anderson et al., 2016; Jiang \& Sisk, 2019). However, while VMAC exhibits these habit-like features, it remains unclear whether VMAC fits the key property of a habit defined in the behavioural neuroscience literature - namely that the conditioned response (attentional prioritisation) occurs independently of changes in the value of the outcome. 


\section{The current study}

To this end, we designed two experiments that incorporated an instructed change in outcome values into a VMAC procedure that was based on the task used by Pearson et al. (2016). Hereafter, this will be referred to as the attentional revaluation task. The key innovation of this task was to introduce 'outcome' elements that mediated between stimuli (colours) and rewards (gain or omission of money). Specifically, the colour of a distractor stimulus signalled the type of fruit that could be won on the current trial, with different fruits having different values. By introducing the mediating fruit outcomes, we were able to keep the relationship between a stimulus and an outcome constant (e.g., a blue distractor always signalled that banana was available) while changing the value of that outcome by manipulating how much money each fruit was worth. This procedure allowed us to examine the effect of these changes in outcome value on patterns of attentional prioritisation of the reward-related distractors.

At the start of each experiment, participants were told that the aim of the task was to earn as many points as possible, since points would later be converted into a cash reward. They were also told that they could earn points by winning fruits, with one type of fruit worth a large number of points (high-value fruit), and the other fruit worth a small number of points (low-value fruit). On each trial of an initial training phase, participants had to make a rapid saccade to a diamond-shaped target among circles to earn a fruit, with the colour of a coloursingleton distractor circle signalling the type of fruit that was available: one colour (the hightraining distractor) signalled that the high-value fruit was available, and the other colour (low-training distractor) signalled the low-value fruit. Following Pearson et al. (2016), if participants looked at the coloured distractor on a given trial, the fruit that could have been earned on that trial was cancelled. In line with previous findings of VMAC, we expected participants to have their attention captured more often by the high-training distractor than 
the low-training distractor, even though this meant that participants would be more likely to miss out on outcomes of greater value. This pattern of results would suggest the high-training distractor stimulus has a greater ability to elicit an attentional prioritisation response, relative to the low-training distractor.

Following this training phase, participants in the not-revalued group were instructed that the values of the fruits that were learned during training would remain the same during a subsequent test phase. By contrast, participants in the revalued group were instructed that the values of each of the fruits had switched - namely, the high-value fruit was thereafter worth a small number of points, and the low-value fruit was worth a large number of points. We then tested the effect of this instructed revaluation manipulation on participants' established pattern of attentional bias. In this test phase, we used a 'nominal extinction' procedure, where participants in both these groups were told that they could still earn fruits, but they were not told about the identity of the fruit they earned on each trial. Nominal extinction is commonly used in tests of habits in humans, as it prevents new learning of relationships between stimuli and outcome values, while preserving participants' motivation to perform (e.g., Luque et al., 2017, 2020; Tricomi et al., 2009; Watson et al., 2018).

This procedure allowed us to examine whether the attentional prioritisation response developed during the training phase was sensitive to changes in the values of fruits following instructed revaluation. If conditioned prioritisation of the high-training distractor had become habit-like - and hence independent of the current value of the associated outcome - then we would expect the pattern learned in training (of greater capture by the high-training distractor than the low-training distractor) to persist in the revalued group during the test phase, despite the reversal of the values of the associated fruit outcomes. By contrast, if prioritisation of the high-training distractor was moderated by a representation of the associated outcome- and hence dependent on the current value of that outcome-we would expect the pattern of 
attentional bias in the revalued group to reflect changed values of the fruit outcomes, with the low-training distractor now more likely to capture attention than the high-training distractor. We could assess any change in the pattern of attentional bias in the revaluation group by comparing against the baseline provided by the non-revalued group, for which we expected the conditioned pattern of greater capture by the high-training distractor to persist during the test phase, as the values of the fruit outcomes remained unchanged from training.

A less interesting reason why attentional biases might not change following outcome revaluation would be if the revaluation manipulation was, for some reason, ineffective in changing the values of the outcomes. In animal studies of habitual behaviour, the effectiveness of revaluation is typically assessed by including an additional group of animals who continue to receive the (now-devalued) outcome during the test phase, such that these animals directly experience pairings of the stimulus (e.g., sight of the lever) and response (pressing the lever) with the devalued outcome (food pellets on which they have been sated). The typical finding is that these animals tested with outcome feedback rapidly cease conditioned responding, confirming that devaluation has indeed been successful in changing the value of the outcome (e.g., Adams, 1982; Adams \& Dickinson, 1981). Following this general approach, we included a third group of participants: the revalued-feedback group. Participants in this group underwent instructed revaluation of the fruit outcomes (as in the revalued group), but they continued to receive trial-by-trial feedback on the identity of the fruit earned on each trial of the test phase. That is, in addition to the knowledge of the revalued fruit outcomes, participants in this group were provided with direct experience of the relationship between distractor colours and now-revalued fruit outcomes. If the instructed revaluation manipulation was effective in changing the values of the fruits, we would expect participants in the revalued-feedback group to show a change in attentional bias in the test phase (relative to the training phase, and to the baseline not-devalued group) such that the 
low-training distractor is now more likely to capture attention than the high-training distractor.

\section{Experiment 1}

\section{Method}

\section{Participants}

Previous studies using variants of the current procedure have found medium to very large effect sizes $\left(d_{z}=0.54-2.20\right)$ for the influence of reward on attentional capture (e.g., Le Pelley et al., 2015; Pearson et al., 2016; Watson et al., 2019a). Consequently we recruited 29 participants per condition; G*Power (with default settings for correlation among repeated measures and nonsphericity correction) revealed that this would give power of .80 to detect a medium-sized effect $\left(d_{z}=0.54\right)$ of reward on attention in each condition, and power $>.95$ to detect a medium-sized effect $\left(\eta_{\mathrm{p}}^{2}=.06\right)$ for the interaction relating to differences in the reward-related attentional bias across conditions. In total, 87 UNSW Sydney students (65 females; age $M=19.52$, $S E M=0.31$ years) participated in this experiment for course credit. Participants also received a monetary bonus at the end of the experiment, which depended on the points they earned in the attentional revaluation task $(M=\mathrm{A} \$ 9.83, S E M=\mathrm{A} \$ 0.23)$. Participants were allocated to one of three groups (not-revalued, revalued, revalued-feedback: $n=29$ per group) in three-way alternation based on their order of arrival. All research reported here was approved by the UNSW Human Research Ethics Advisory Panel (Psychology); experiment scripts and raw data are available at https://osf.io/nuaxg.

\section{Apparatus}

Stimuli were presented on a 23 -inch monitor $(60 \mathrm{~Hz}$ refresh rate, $1920 \times 1080$ resolution); stimulus presentation was controlled by MATLAB with Psychophysics Toolbox extensions (Brainard, 1997; Pelli, 1997; Kleiner, Brainard \& Pelli, 2007). Monitor-mounted 
eye-trackers were used to record eye movements in the attentional revaluation task. For the first 34 participants, eye-tracking was via Tobii TX300 eye-trackers (sampling rate $300 \mathrm{~Hz}$ ). These trackers were upgraded part-way through the testing period, with subsequent participants tested using Tobii Pro Spectrum eye-trackers (sampling rate $600 \mathrm{~Hz}$ ). For all participants, gaze data were down-sampled to $100 \mathrm{~Hz}$ for gaze-contingent calculations during stimulus presentation. Preliminary analyses indicated that the model of eye tracker had no significant effect on the pattern of results, and hence we collapsed across this factor in all analyses reported here. Each participant's head position was stabilised using a chin-rest $60 \mathrm{~cm}$ from the monitor. The eye-tracker was calibrated at the start of the experiment using a 5-point procedure.

\section{Design and procedure}

The attentional revaluation task consisted of four components: the initial value instructions, training phase, revaluation instructions, and test phase.

Initial value instructions. At the start of the experiment, participants were told that their aim should be to earn points, since these points would be converted into cash at the end of the session, and that they could win points by earning fruits - lemons and bananas. Half of the participants were initially informed that lemons were worth 500 points and bananas were worth 10 points (Figure 1A); for the other half of participants, this was reversed. Participants were told that they could earn these fruits by moving their eyes to the diamond target on each trial "as quickly and as directly as possible", but that if they looked at the coloured circle that was present in the search display, the fruit they could have earned would be cancelled.

However, participants were not informed of the specific colour-fruit contingencies (e.g., that a blue circle signalled availability of lemons and an orange circle signalled bananas). 


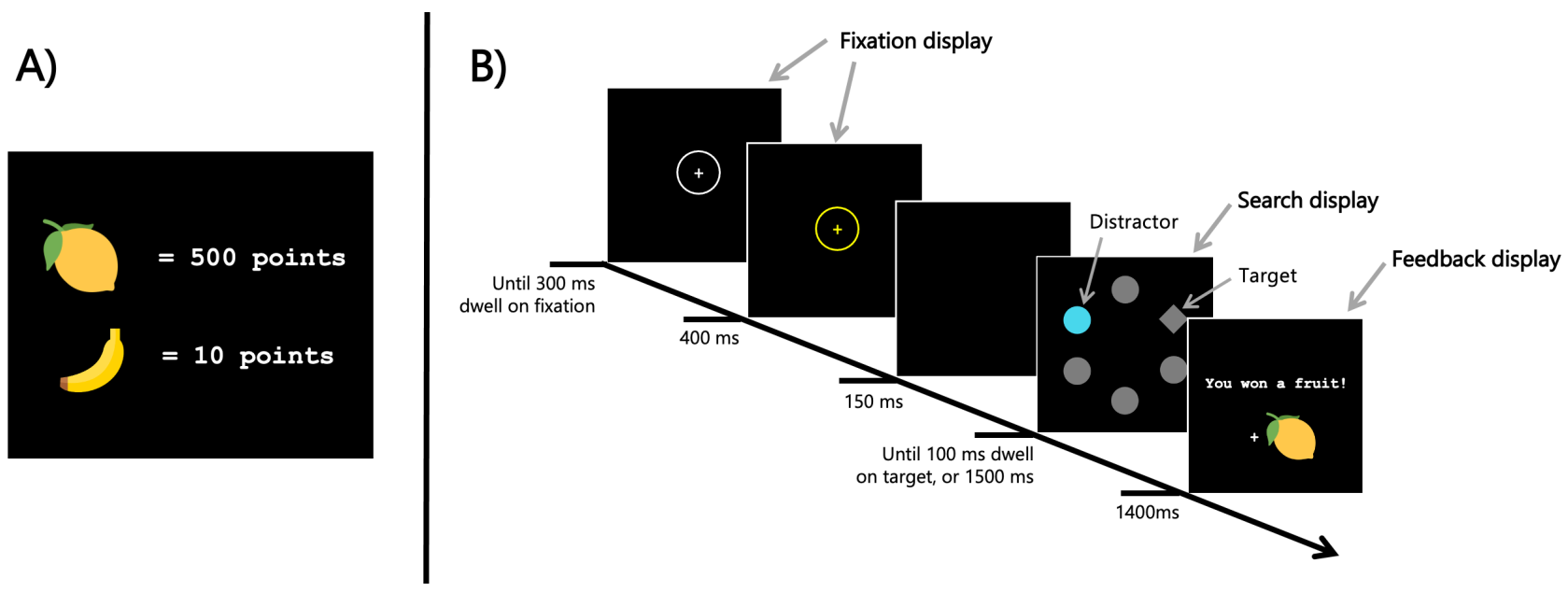

Figure 1. Examples of stimulus presentation. A) Participants were informed of the values of each fruit outcome at the start of the attentional revaluation task, and reminded of these values prior to each block of trials. B) An example trial. On each trial, participants first focussed on a central fixation cross. A search display then appeared, containing a diamond-shaped target among circles. One of these circles was a colour-singleton distractor, whose colour signalled the type of fruit available for making a rapid saccade to the target. The example here shows a high-training distractor trial, in which a blue distractor signals availability of a fruit (lemon in this case) that is worth 500 points; note that fruit-value and colour-fruit contingencies were counterbalanced across participants. If participants looked at the distractor before looking at the diamond, or if they did not respond quickly enough, the fruit reward would not be delivered on that trial.

Training phase. Each trial of the search task consisted of a fixation display, search display, and feedback display (Figure 1B). All stimuli were presented on a black background. The fixation display comprised a central white cross $\left(0.5^{\circ}\right.$ visual angle $)$ surrounded by a white circle (diameter $3.0^{\circ}$ ). When participants had accumulated $700 \mathrm{~ms}$ of gaze time inside this circle, or after $4000 \mathrm{~ms}$, the cross and circle turned yellow to indicate that the search display was imminent. After $300 \mathrm{~ms}$ the screen blanked, and after a 150-ms delay the search display appeared: a diamond and 5 circles, each $2.3 \times 2.3^{\circ}$ visual angle, distributed evenly around the centre of the screen, with the centre of each shape at an eccentricity of $5.1^{\circ}$. One of the circles was coloured either orange (CIE $x, y$ chromaticity coordinates $.493 / .445$ ) or blue 
(CIE $x, y .192 / .216)$ with similar luminance $\left(\sim 24.5 \mathrm{~cd} / \mathrm{m}^{2}\right)$ : we term this colour-singleton circle the distractor. All other shapes were grey (CIE $x, y .327 / .400$, luminance $\sim 8.3 \mathrm{~cd} / \mathrm{m}^{2}$ ).

Participants' task was to move their eyes to the diamond target as quickly as possible; a response was registered when they had accumulated $100 \mathrm{~ms}$ of gaze dwell time within a region of diameter $3.5^{\circ}$ centred on this target. The colour of the distractor in the search display signalled the type of fruit that was available for a rapid response: for half of the participants, a blue distractor signalled that a rapid saccade to the diamond target would earn a lemon, and an orange distractor signalled a banana; for remaining participants this assignment was reversed. The distractor signalling the high-value fruit (worth 500 points) was termed the high-training distractor, and the distractor signalling the low-value fruit (worth 10 points) was the low-training distractor. If any gaze fell on or near the distractor (within a region of diameter $5.1^{\circ}$ centred on this distractor) prior to a response being registered, no reward was given on that trial; these were recorded as distraction trials.

The search display was replaced by the feedback display immediately when a response was registered, or after $2000 \mathrm{~ms}$ (timeout). If response time was less than $1000 \mathrm{~ms}$ and it was not a distraction trial, then the feedback display stated "Fruit won!", and showed a picture of the appropriate fruit. If the trial was a distraction trial, feedback stated "No reward: You could have won:", and showed the fruit overlaid with a large red ' $\mathrm{X}$ '. If response time was greater than $1000 \mathrm{~ms}$, feedback stated "Too slow: You could have won:", and presented the fruit overlaid with a red ' $X$ '. If no response was registered before the trial timed-out, feedback read: "Too slow: Please try to look at the diamond more quickly". Feedback appeared for $1400 \mathrm{~ms}$; the next trial then began after a blank inter-trial interval of $1400 \mathrm{~ms}$.

Participants completed 16 blocks of trials in the training phase, with each block containing 24 trials (384 trials in total). Half of the trials in each block featured a hightraining distractor, and the other half featured a low-training distractor: trial order in each 
block was random. The locations of the target and distractor were randomly determined on each trial. At the end of each block participants took a short break, during which they were informed of the total number of points they had earned so far. During each break, participants also received a reminder of the fruit values (as in Figure 1A) which appeared on the screen for at least 10s; following this 10 s period, participants opted when to continue with the task.

Revaluation and feedback instructions. Immediately following the training phase, participants received further instructions depending on their group assignment (summarised in Table 1) - the not-revalued group (hereafter called the NoRev group), the revalued group (Rev group), and the revalued with feedback group (RevFB group) - with different groups receiving different instructions about the values of the two fruits, and/or the feedback structure. Participants in the NoRev group were reminded of the values of the fruits, which were unchanged from training (e.g., if lemons had previously been worth 500 points and bananas 10 points, participants in the NoRev group were simply reminded of these relationships). By contrast, participants in the Rev and RevFB groups were told that the values of the fruits had changed, such that the fruit previously worth 500 points in training was now worth 10 points, and the fruit previously worth 10 points was now worth 500 points. After this instruction, all participants answered check questions to ensure their knowledge of the current fruit values: participants were shown a picture of a lemon and a banana and asked to select the current value of each fruit. Both responses were required to be correct before they could proceed. 
Table 1. Design of each phase for one counterbalance condition. Colours refer to colours of the distractor in the search display; fruits refer to outcomes that could be won for rapid responses. Note: fruit-value and colour-fruit contingencies were counterbalanced across participants.

\begin{tabular}{|c|c|c|c|c|}
\hline Group & $\begin{array}{l}\text { Initial value } \\
\text { instructions }\end{array}$ & Training phase & $\begin{array}{l}\text { Revaluation } \\
\text { instructions }\end{array}$ & Test phase \\
\hline NoRev & \multirow{3}{*}{$\begin{array}{l}\text { Lemon }=500 \mathrm{pts} \\
\text { Banana }=10 \mathrm{pts}\end{array}$} & \multirow{3}{*}{$\begin{array}{l}\text { Blue } \rightarrow \text { Lemon } \\
\text { Orange } \rightarrow \text { Banana }\end{array}$} & $\begin{array}{l}\text { Lemon }=500 \mathrm{pts} \\
\text { Banana }=10 \mathrm{pts}\end{array}$ & $\begin{array}{l}\text { Blue } \rightarrow ? ? \\
\text { Orange } \rightarrow ? ?\end{array}$ \\
\hline $\operatorname{Rev}$ & & & $\begin{array}{l}\text { Lemon }=10 \mathrm{pts} \\
\text { Banana }=500 \mathrm{pts}\end{array}$ & $\begin{array}{l}\text { Blue } \rightarrow ? ? \\
\text { Orange } \rightarrow ? ?\end{array}$ \\
\hline RevFB & & & $\begin{array}{l}\text { Lemon }=10 \mathrm{pts} \\
\text { Banana }=500 \mathrm{pts}\end{array}$ & $\begin{array}{l}\text { Blue } \rightarrow \text { Lemon } \\
\text { Orange } \rightarrow \text { Banana }\end{array}$ \\
\hline
\end{tabular}

In addition, participants in the NoRev and Rev groups were instructed that they would no longer be told whether they had earned a lemon or a banana on each trial; "instead you will simply be told whether or not you won a fruit - and we will keep track of how many points you have earned." By contrast, participants in the RevFB group were told that "as before, you will be told whether you earned a lemon or a banana on each trial, and we will keep track of how many points you have earned".

Test phase. All participants then completed the test phase of the search task. In the test phase, participants in the Rev and NoRev groups continued to earn fruits for responding correctly, but did not receive feedback on the identity of the fruit earned on each trial. If response time was below $1000 \mathrm{~ms}$ and it was not a distraction trial, then feedback stated "Fruit won!", accompanied by “??” where previously a picture of the fruit had appeared during the training phase. If the trial was a distraction trial, feedback stated "No reward: You could have won a fruit", and displayed "??” overlaid with a red ' $\mathrm{X}$ '. If response time was above 1000 ms, feedback stated "Too slow: You could have won a fruit", and displayed "??" overlaid with a red ' $\mathrm{X}$ '. Participants in the RevFB group received feedback on the identity of the fruit earned — or omitted—on each trial (lemon or banana), as in the training phase. 
Participants completed 8 blocks of trials in the test phase, with blocks structured as in the training phase. Participants in all groups were reminded of the current values of each fruit in the short break that followed each block.

Knowledge checks. Following the test phase, participants' knowledge of the colourfruit contingencies was assessed (i.e., whether participants had learned that a particular distractor colour signalled the availability of a particular type of fruit). Participants were told that the type of fruit that could be won on each trial depended on the colour of the coloured circle in the search display. They were then presented with an orange and a blue circle, in random order, and for each they were asked to select which fruit (banana or lemon) they could win when that stimulus appeared in the search display.

\section{Data preparation}

Data analysis followed our established protocols (e.g., Le Pelley et al., 2015; Pearson et al., 2015, 2016; Watson et al., 2019). We discarded data from the first two trials after each break, trials that timed out with no response ( $1.46 \%$ of all trials), and trials with less than $25 \%$ valid gaze data (as a result of blinks, poor eye-tracking etc.: $0.42 \%$ of trials). For the remaining trials, valid gaze data were registered in $99.0 \%(S E M=0.004 \%)$ of samples from the eye-tracker.

The main dependent variable of interest was the proportion of distraction trials: the proportion of trials on which participants looked at the coloured distractor, resulting in cancellation of the fruit outcome. We analysed proportion of distraction trials as a function of whether the trial featured a high- or low-training distractor; note that we label distractors according to the value they signalled during the training phase.

\section{Results}

We first examined the proportion of distraction trials across the training and test phases using a 2 (phase: training vs. test phase) $\times 2$ (distractor-type: high- vs. low-training 
distractor) $\times 2$ (group: NoRev, Rev, RevFB) ANOVA. Importantly, the three-way interaction was significant, $F(1,84)=10.94, p<.001, \eta_{\mathrm{p}}^{2}=.21$, suggesting a significant difference in the distractor-type $\times$ group interaction between the training and test phases. We note that this analysis averages training phase performance across all blocks of training, including the initial training blocks where the effect of reward may have been relatively small (as participants had not yet had substantial exposure to the reward contingencies). Hence, we repeated this ANOVA, restricting data from the training phase to only the last two blocks immediately prior to the value-switch manipulation - this did not affect the pattern of significant findings (see Supplementary Materials). Planned follow-up analyses focused on the proportion of distraction trials in the training and test phases separately.

\section{Training phase}

We first analysed proportion of distraction trials during the training phase, before the revaluation manipulation, using a 2 (distractor-type: high- vs. low-training distractor) $\times 3$ (revaluation group: NoRev, Rev, RevFB) ANOVA (the analysis reported here collapses across the whole training phase; analysis restricted to the final two blocks is reported in Supplementary Materials). There was a significant main effect of distractor-type, $F(1,84)=$ $54.19, p<.001, \eta_{\mathrm{p}}^{2}=.39$, with a greater proportion of distraction trials when the display featured a high-training than a low-training distractor (Figure 2A). That is, participants were more likely to look at the distractor signalling availability of a high-value fruit than the distractor signalling a low-value fruit, even though this pattern of behaviour was counterproductive because looking at the distractor caused cancellation of the fruit. There was no significant main effect of revaluation group, $F(2,84)=0.12, p=.89, \eta_{\mathrm{p}}{ }^{2}=.003$, nor was there a revaluation group $\times$ distractor-type interaction, $F(2,84)=0.30, p=.75, \eta_{\mathrm{p}}{ }^{2}=.01$. These null findings are unsurprising, since all groups received equivalent treatment until after the training phase. 
A) Training Phase

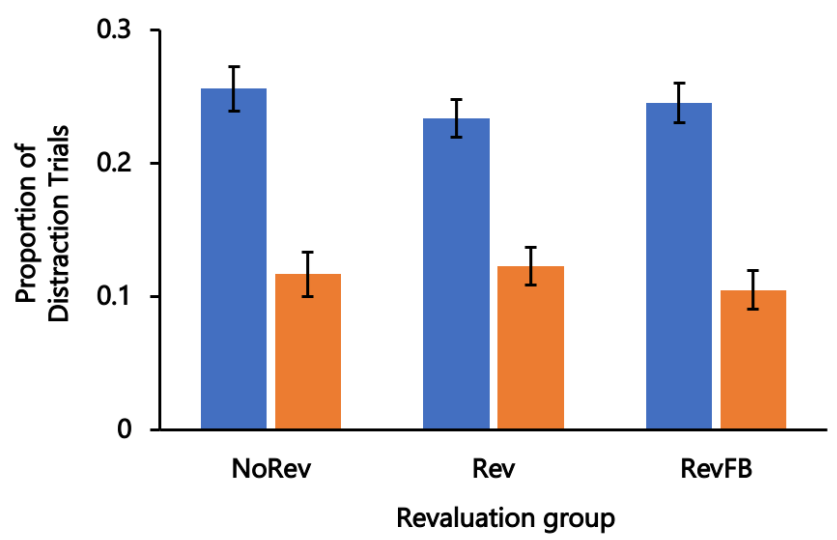

- High-training distractor

- Low-training distractor

B) Test phase

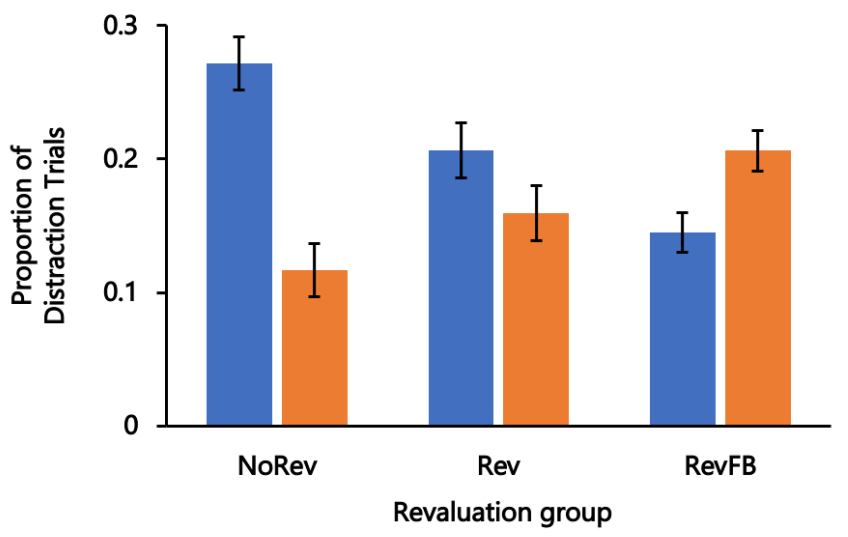

C)

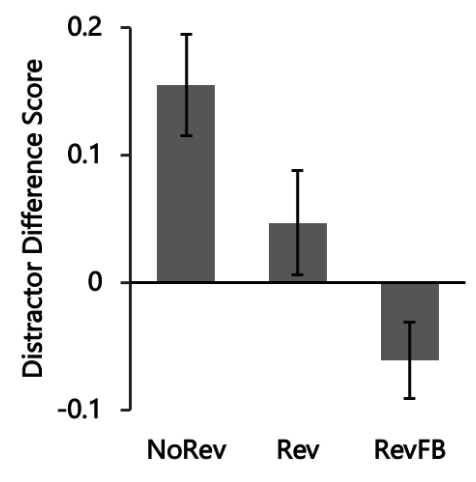

Figure 2. Proportion of distraction trials for trials with high- and low-training distractors in A) the training phase and B) the test phase of Experiment 1. Distraction trials occurred when participants looked at the coloured distractor in the search display, resulting in cancellation of the fruit outcome. Note that distractors are defined by the value of the outcome they signalled during the training phase (e.g., a high-training distractor was the colour associated with a high-value fruit outcome during training). Panel C) shows distractor difference scores (given by the difference in the mean proportion of distraction trials between high- versus low-training distractor trials) for each revaluation group during the test phase. Error bars in Panels A and B show withinsubjects SEM (Morey, 2008), and in Panel C show between-subjects SEM.

\section{Test phase}

Figure $2 \mathrm{~B}$ shows the proportion of distraction trials for each revaluation group in the test phase, following the instructions regarding revaluation of the fruit outcomes. These data were analysed using a 2 (distractor-type) $\times 3$ (revaluation group) ANOVA. This revealed a 
significant main effect of distractor-type, $F(1,84)=4.77, p=.032, \eta_{\mathrm{p}}{ }^{2}=.05$, while the main effect of revaluation group was not significant, $F(2,84)=0.26, p=.78, \eta_{\mathrm{p}}^{2}=.006$. Critically, we found a significant interaction between distractor-type and revaluation-group, $F(2,84)=$ $8.38, p<.001, \eta_{\mathrm{p}}{ }^{2}=.17$, indicating that the pattern of attentional bias differed across the three groups during the test phase.

To decompose this interaction, we first examined the simple effect of distractor-type in each group using paired-samples $t$-tests. In the NoRev group, there was a significant effect of distractor-type, $t(28)=3.89, p<.001, d_{z}=0.72$, with participants more likely to look at the high-training distractor than the low-training distractor (difference scores are shown in Figure $2 \mathrm{C})$. By contrast, in the Rev group, there was no significant effect of distractor type, $t(28)=$ $1.14, p=.26, d_{z}=0.21$. Finally, in the RevFB group, participants were more likely to look at the low-training distractor than the high-training distractor, though this difference only approached significance, $t(28)=2.04, p=.051, d_{z}=0.38$.

We then compared between-group differences in attentional capture by examining the interaction between revaluation group and distractor-type. First, the Rev group and NoRev group differed only in whether or not the values of the fruits changed from the training phase to the revaluation phase (neither group received feedback on the specific fruit won on each trial during the revaluation phase). Hence, differences in patterns of attentional capture between these two groups during the test phase provided an index of the effect of outcome revaluation on attentional capture. A mixed $2 \times 2$ ANOVA revealed a significant main effect of distractor-type, $F(1,56)=12.45, p<.001, \eta_{\mathrm{p}}{ }^{2}=.182$, while the main effect of revaluation group was not significant, $F(1,56)=0.22, p=.64, \eta_{\mathrm{p}}^{2}=.004$. Notably, the interaction between revaluation group and distractor-type approached, but did not reach, significance, $F(1,56)=3.56, p=.064, \eta_{\mathrm{p}}{ }^{2}=.06$, with a trend towards a greater difference in capture by 
high-training versus low-training distractors in the NoRev group than the Rev group (Figure $1 C)$.

Our second comparison of interest was between the Rev and RevFB groups. These groups differed only in whether or not trial-by-trial feedback on the specific fruit outcomes was provided during the test phase (both groups experienced the revaluation of fruit outcomes). Hence, any difference in patterns of attentional capture between these two groups provided an index of the effect of feedback following outcome revaluation. A $2 \times 2$ ANOVA revealed that the main effect of distractor-type was not significant, $F(1,56)=0.08, p=.78$, $\eta_{\mathrm{p}}{ }^{2}=.001$, nor was the main effect of revaluation group, $F(1,56)=.07, p=.79, \eta_{\mathrm{p}}{ }^{2}=.001$. However, the interaction between revaluation group and distractor-type was significant, $F(1,56)=4.52, p=.038, \eta_{\mathrm{p}}^{2}=.075$, with participants in the RevFB group exhibiting a greater difference in attentional capture by high- versus low-training distractors than participants in the Rev group (Figure 1C).

Finally, a $2 \times 2$ ANOVA comparing the NoRev and RevFB groups revealed no main effect of distractor-type, $F(1,56)=3.56, p=.065, \eta_{\mathrm{p}}^{2}=.060$, or revaluation group, $F(1,56)=$ $0.48, p=.49, \eta_{\mathrm{p}}^{2}=.009$, but a highly significant interaction, $F(1,56)=18.78, p<.001, \eta_{\mathrm{p}}^{2}=$ .251 , in line with the very different pattern of attentional bias observed during the test phase in these groups (Figure 1C).

\section{Colour-fruit knowledge checks}

We conducted analyses on participants' knowledge of colour-fruit contingencies, which was tested at the end of the experiment. In each of the three groups, 26 out of 29 participants reported the correct colour-fruit associations.

We repeated the previously described analyses of proportion of distraction trials while excluding the nine participants who failed to correctly identify the colour-fruit contingencies. These analyses did not affect the pattern of significant findings, with the exception that the 
pattern of attentional bias in the test phase now differed significantly between Rev and NoRev groups $\left(F(1,50)=6.22, p=.016, \eta_{\mathrm{p}}{ }^{2}=.111\right)$, where previously this difference had only approached significance $(p=.064)$.

\section{Discussion}

Experiment 1 examined the effect of switching the values of outcomes on attentional prioritisation of distractor stimuli. In the training phase, prior to the revaluation manipulation, participants in all three groups were more likely to have their attention captured by a distractor signalling a high-value fruit outcome versus a low-value fruit. This finding is consistent with previous demonstrations of value-modulated attentional capture (e.g., Le Pelley et al., 2015; Pearson et al., 2016), wherein the size of the reward associated with a stimulus modulates the extent to which that stimulus captures attention. Viewed another way, findings from the training phase suggest that the high-training distractor stimulus was more likely to elicit an attentional prioritisation response than the low-training distractor.

We were particularly interested in whether the three groups differed in patterns of attentional prioritisation in the test phase of the attentional revaluation task. For participants in the NoRev group, there was no change in the value of the fruit outcomes between training and test phases: these participants were instructed that the fruit that had high value in the training phase continued to have high value in the test phase, and vice versa. Participants in this NoRev group continued to show greater attentional capture by the high-training distractor than the low-training distractor, even though no feedback was provided as to the specific fruit earned on each trial of the test phase. Thus for group NoRev, the attentional bias established during the training phase persisted during the test phase, even in the absence of further feedback (cf. Watson et al., 2019).

Crucially, for participants in the Rev group, the values of the fruit outcomes switched between the training and test phases; the fruit that had high value in the training phase 
thereafter had low value, and the fruit that had low value in the training phase had high value. During the test phase, these participants no longer exhibited significantly greater attentional capture by the high-training distractor than the low-training distractor. This finding is consistent with the idea that the instructed value-switch produced a change in attentional bias relative to training, and could be interpreted to suggest that the attentional bias developed during training was (at least to some degree) mediated by a representation of the outcome and its current value. Some caution is warranted in drawing this conclusion, given that the difference between NoRev and Rev groups in the pattern of attentional bias during the test phase did not reach significance in the full sample $(p=.064)$. However, and consistent with the idea that attentional prioritisation was sensitive to changes in reward values, when we excluded participants who failed the explicit knowledge check regarding stimulus-outcome relationships, the Rev group showed a significantly smaller difference in attentional capture by the high-training versus low-training distractor than the NoRev group.

The difference in test-phase performance between Rev and NoRev groups suggests that the attentional prioritisation response conditioned during the training phase was at least partially mediated by a representation of the current value of the outcome associated with each type of distractor. That said, behaviour in the Rev group was not purely outcomemediated. Participants in this group were aware of the new values of the fruits in the test phase, since they were explicitly told this information, reminded of it repeatedly, and required to pass check questions on the values of the fruits following revaluation. Moreover, almost all participants in the Rev group were aware of which fruit outcome was associated with each colour of distractor. Yet, in the test phase, attentional prioritisation of the high-training distractor was not completely overcome to follow the changed values of the fruits. This conclusion is supported by analysis of the test-phase data from the Rev group using a Bayesian $t$-test (using the default prior in JASP: JASP Team, 2020) to assess evidence for the 
one-tailed alternative hypothesis of greater capture by the low-training distractor than the high-training distractor (the pattern expected if attention were purely mediated by current outcome value). This analysis yielded a Bayes factor of $B F_{01}=9.97$, representing substantial evidence in favour of the null hypothesis over this alternative (Jeffreys, 1961). This finding suggests that the attentional prioritisation response conditioned during the training phase was not completely mediated by representations of the outcomes and their current values.

Like the Rev group, participants in the RevFB group also experienced a switch in the values of the fruit outcomes between the training and test phases. Notably, participants in the RevFB group also received feedback as to the specific fruit earned on each trial of the test phase. In the test phase, participants in the RevFB group no longer exhibited significantly greater attentional capture by the high-training distractor than the low-training distractor, as they had in the training phase. In fact, there was a trend for the reverse pattern; these participants showed greater attentional capture by the low-training distractor than the hightraining distractor following the revaluation manipulation, with this difference closely approaching significance $(p=.051)$. Certainly the pattern of test-phase performance in the RevFB group was very different from the baseline provided by the NoRev group (Figure 1C), and this difference shows that the instructed outcome devaluation manipulation was effective in changing participants' beliefs about the values of the fruit outcomes (cf. Adams, 1982). Thus the failure to observe a full reversal of attentional bias in the Rev group cannot easily be attributed to ineffective outcome revaluation.

There was a significant difference in test-phase performance between the Rev and RevFB groups. Both of these groups underwent outcome revaluation; the only difference was that participants in the RevFB group continued to receive trial-by-trial feedback on the identity of the specific fruit earned on each trial of the test phase, whereas the Rev group did not. Although participants in the Rev group had knowledge of the new values of the fruit 
outcomes, and knowledge of which fruit outcome was associated with each distractor, this knowledge alone was not sufficient to overcome the attentional bias toward the high-training distractor. Only with the additional experience of the pairings of distractor colours and revalued outcomes was a full reversal of the attentional prioritisation response toward highand low-training distractors observed (in the RevFB group) - consistent with the idea that attentional biases in this task are not purely mediated by knowledge of current outcome values, but instead must be learned based on experience of pairings of stimuli, outcome identities, and associated values.

Interestingly, it should be noted that for participants in the Rev and RevFB groups, continued attentional prioritisation of the high-training distractor (over the low-training distractor) during the test phase would have been advantageous, as it would have resulted in the cancellation of only a low-value fruit. Instead, participants in both groups no longer showed an attentional bias towards the high-training distractor during the test phase.

Strikingly, participants in the RevFB group tended to attentionally prioritise the low-training distractor, resulting in the cancellation of high-value fruits. These findings raise the idea that motivated efforts to earn the high value fruit under the changed value conditions were met with a corresponding - yet counterproductive - change in attentional prioritisation.

\section{Experiment 2}

Experiment 2 investigated how motivation would influence the sensitivity of the attentional prioritisation response to changes in outcome values. In the test phase of Experiment 1, participants in the Rev and RevFB groups could still earn a large reward, since one of the fruits was worth 500 points). Hence, any shifts in patterns of attentional prioritisation following revaluation may have occurred in a state of high motivation to earn a large reward (although paradoxically, these shifts would have reduced the likelihood of earning that reward). In Experiment 2a, we instead used an outcome devaluation 
manipulation to examine whether or not patterns of attentional prioritisation would shift even if a large reward could no longer be earned in the test phase - that is, even if motivation to earn fruit outcomes was presumably low. Conversely, in Experiment 2b, we used a supervaluation manipulation to examine whether patterns of attentional prioritisation would change if both outcomes were associated with large rewards - that is, if motivation to earn both outcomes was presumably high.

The training phases of Experiment $2 \mathrm{a}$ and $2 \mathrm{~b}$ were as in Experiment 1: participants learned that one distractor signalled a high-value fruit (worth 500 points) and the other distractor signalled a low-value fruit (10 points). We again expected that participants' attention would be captured more often by the high-training distractor than the low-training distractor, consistent with the idea that the high-training distractor was imbued with a greater ability to elicit an attentional prioritisation response than the low-value distractor.

Following the training phase of Experiment 2a, participants were allocated to either the devaluation (Dev) group or the devaluation-with-feedback (DevFB) group. Participants in both groups were informed that the high-value fruit was now devalued, such that both fruits were thereafter worth only 10 points. Both groups then completed the test phase, with feedback on fruit-outcome identity provided for the DevFB group but not the Dev group. ${ }^{1}$ Following the training phase of Experiment 2b, participants were allocated to either the super-valued (Super) group or the super-valued-with-feedback (SuperFB) group. Participants in both groups were informed that the low-value fruit had increased in value, such that both fruits were thereafter worth 500 points. Both groups then completed the test phase, with trialby-trial feedback on the fruit-outcome identity provided only to the SuperFB group.

\footnotetext{
${ }^{1}$ Experiments $2 \mathrm{a}$ and $2 \mathrm{~b}$ did not include a group for whom outcome values remained unchanged in the test phase. Such a group would have been identical to the NoRev group of Experiment 1, and hence was omitted in order to save resources.
} 
As in Experiment 1, we were interested in whether or not conditioned attentional prioritisation of the high-training distractor that developed during the training phase would be sensitive to changes in the values of outcomes. If conditioned attentional responses were indeed mediated by representations of the fruit outcomes (and their new, equal values), then the attentional bias formed in the training phase should disappear in the test phase. If instead conditioned attentional responses had become 'habit-like', operating independently of representations of the outcomes, then we would expect the attentional bias toward the hightraining distractor to persist in the test phase.

Performance of participants in the Dev and Super groups (Experiments $2 \mathrm{a}$ and $2 \mathrm{~b}$ respectively) allowed us to examine whether knowledge of the new outcome values alone was sufficient to induce a change in attentional bias. Performance of participants in the DevFB and SuperFB groups allowed us to assess whether any change in attentional bias in the test phase additionally required direct experience of pairings of distractor stimuli with newly devalued outcomes.

\section{Method}

\section{Participants and apparatus}

Fifty-one UNSW Sydney students (31 females; age $M=18.56, S E M=0.19$ years) participated in Experiment 2a and 55 UNSW Sydney students (39 females; age $M=19.18$, $S E M=0.39$ years) participated in Experiment $2 \mathrm{~b}$. All participants earned course credit, and received a monetary bonus dependent on their performance in the attentional revaluation task (Experiment 2a $M=\mathrm{A} \$ 9.67, S E M=\mathrm{A} \$ 0.33$; Experiment 2b $M=\mathrm{A} \$ 9.95, S E M=\mathrm{A} \$ 0.18$ ). Participants in Experiment 2a were allocated into either the Dev group $(n=26)$ or the DevFB group $(n=25)$ in an alternating fashion depending on their order of arrival. Participants in Experiment $2 \mathrm{~b}$ were allocated into either the Super group $(n=28)$ or the SuperFB group $(n=$ 
27) in the same fashion. Apparatus was as for Experiment 1; all participants were tested using

a Tobii Pro Spectrum eye-tracker (sampling rate $600 \mathrm{~Hz}$ ).

\section{Design and procedure}

The training phase was as for Experiment 1. Following the training phase, all participants were told that the values of the fruits had changed (see Table 2). In Experiment 2a, the fruit previously worth 500 points in training was now worth 10 points, while the fruit previously worth 10 points was still worth 10 points. In Experiment $2 b$, the fruit previously worth 10 points in training was now worth 500 points, while the fruit previously worth 500 points was still worth 500 points. Check questions were used to ensure that participants had understood these instructions: participants were required to select the correct current value of each fruit before they could proceed.

Table 2. Design of each phase for one counterbalance condition. Colours refer to colours of the distractor in the search display; fruits refer to outcomes that could be won for rapid responses. Note: fruit-value and colour-fruit contingencies were counterbalanced across participants.

\begin{tabular}{|c|c|c|c|c|c|}
\hline Experiment & Group & $\begin{array}{l}\text { Initial value } \\
\text { instructions }\end{array}$ & Training phase & $\begin{array}{l}\text { Revaluation } \\
\text { instructions }\end{array}$ & Test phase \\
\hline \multirow{2}{*}{$\begin{array}{l}\text { Experiment } \\
2 \mathrm{a}\end{array}$} & Dev & \multirow{2}{*}{$\begin{array}{l}\text { Lemon }=500 \mathrm{pts} \\
\text { Banana }=10 \mathrm{pts}\end{array}$} & \multirow{2}{*}{$\begin{array}{l}\text { Blue } \rightarrow \text { Lemon } \\
\text { Orange } \rightarrow \text { Banana }\end{array}$} & \multirow{2}{*}{$\begin{array}{l}\text { Lemon }=10 \mathrm{pts} \\
\text { Banana }=10 \mathrm{pts}\end{array}$} & $\begin{array}{l}\text { Blue } \rightarrow ? ? \\
\text { Orange } \rightarrow ? ?\end{array}$ \\
\hline & DevFB & & & & $\begin{array}{l}\text { Blue } \rightarrow \text { Lemon } \\
\text { Orange } \rightarrow \text { Banana }\end{array}$ \\
\hline \multirow{2}{*}{$\begin{array}{l}\text { Experiment } \\
\text { 2b }\end{array}$} & Super & \multirow{2}{*}{$\begin{array}{l}\text { Lemon }=500 \mathrm{pts} \\
\text { Banana }=10 \mathrm{pts}\end{array}$} & \multirow{2}{*}{$\begin{array}{l}\text { Blue } \rightarrow \text { Lemon } \\
\text { Orange } \rightarrow \text { Banana }\end{array}$} & \multirow{2}{*}{$\begin{array}{l}\text { Lemon }=500 \mathrm{pts} \\
\text { Banana }=500 \mathrm{pts}\end{array}$} & $\begin{array}{l}\text { Blue } \rightarrow ? ? \\
\text { Orange } \rightarrow ? ?\end{array}$ \\
\hline & SuperFB & & & & $\begin{array}{l}\text { Blue } \rightarrow \text { Lemon } \\
\text { Orange } \rightarrow \text { Banana }\end{array}$ \\
\hline
\end{tabular}

Participants in the Dev group (Experiment 2a) and Super group (2b) were further informed that while they would still be earning fruits during the subsequent test phase, they would no longer be told the identity of the fruit earned on each trial. Participants in the 
DevFB and SuperFB groups were informed that they would continue to be told whether they had earned a lemon or a banana on each trial. In other respects, the test phase was as in Experiment 1.

Following the test phase, participants completed a knowledge check assessing their understanding of which fruit was signalled by each colour of distractor (i.e., the colour-fruit contingencies, as in Experiment 1). Moreover, while all participants were required to pass check questions on the current values of each fruit to progress to the test phase, we added a final knowledge check for the fruit-value contingency to further ensure that participants had encoded knowledge of the new fruit-value contingencies. In this latter knowledge check, participants were presented with each fruit in random order and were asked to select whether it was currently worth 500 points or 10 points.

\section{Data preparation}

Data preparation was as in Experiment 1. We discarded the first two trials after each break, time-out trials ( $0.57 \%$ of all trials in Experiment $2 \mathrm{a} ; 1.07 \%$ in Experiment $2 \mathrm{~b}$ ), and trials with less than $25 \%$ valid gaze data $(0.55 \%$ of trials in Experiment $2 \mathrm{a} ; 0.03 \%$ in Experiment 2b). In both experiments, valid gaze data were registered on over $99 \%$ of the gaze-location samples in remaining trials.

\section{Results}

As in Experiment 1, analyses reported in the main text used data collapsed across all trials of the training phase. In Supplementary Materials we report results of analyses in which training-phase data were restricted to only the final two blocks, in order to examine the 
attentional bias immediately prior to the value-switch manipulation - this change did not affect the pattern of significant findings.

\section{Experiment $2 a$}

We first examined the proportion of distraction trials across the training and test phases using a 2 (phase: training vs. test phase) $\times 2$ (distractor-type: high- vs. low-training distractor) $\times 2$ (group: Dev vs. DevFB) ANOVA. Notably, the three-way interaction was not significant, $F(1,49)=0.004, p=.95, \eta_{\mathrm{p}}{ }^{2}<.001$. Nevertheless, planned follow-up analyses focused on the proportion of distraction trials in the training and test phases separately.

Training phase. Figure 3A shows proportion of distraction trials during the training phase. We analysed these data using ANOVA with factors of distractor-type and group. There was a significant main effect of distractor-type, $F(1,49)=26.72, p<.001, \eta_{\mathrm{p}}{ }^{2}=.35$, with more distraction trials when the display contained a high-training distractor than a lowtraining distractor. There was no significant main effect of group, $F(1,49)=1.64, p=.21, \eta_{\mathrm{p}}^{2}$ $=.03$, or distractor-type $\times$ group interaction, $F(1,49)=1.57, p=.22, \eta_{\mathrm{p}}{ }^{2}=.03$. 


\section{A) Training phase}

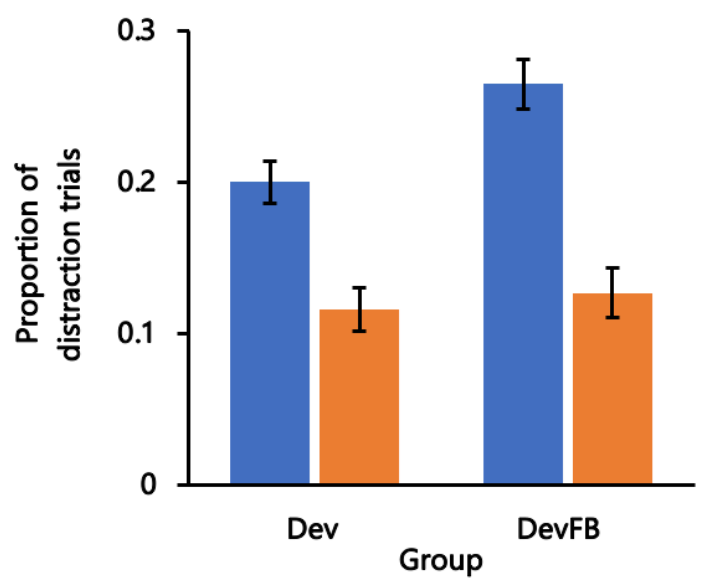

- High-training distractor

- Low-training distractor

B) Test phase

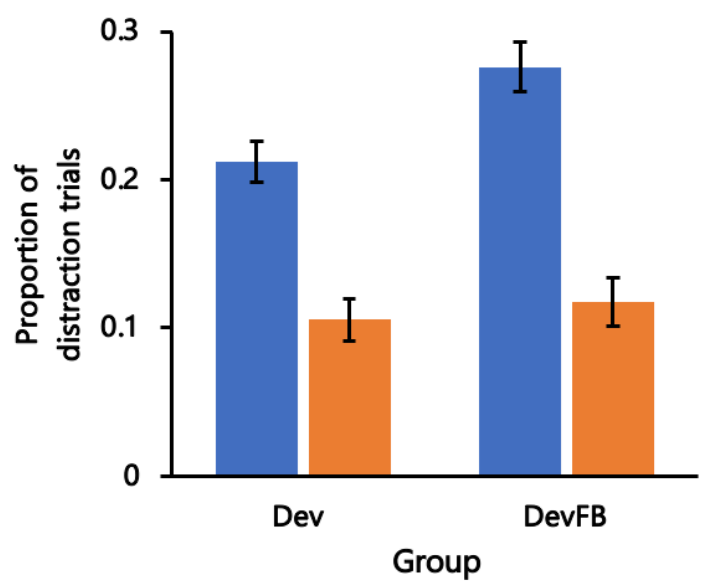

Figure 3. Proportion of distraction trials for trials featuring high- and low-training distractors in A) the training phase and B) the test phase of Experiment 2a. Distraction trials occurred when participants looked at the coloured distractor in the search display, resulting in omission of the fruit outcome on that trial. Note that distractors are defined by the value of the outcome they signalled during the training phase (e.g., a hightraining distractor was the colour associated with a high-value fruit outcome during training). Error bars show within-subjects SEM (Morey, 2008).

Test phase. Figure 3B shows the proportion of distraction trials for each group in the test phase, following the devaluation of the high-value fruit. These data were again analysed using a distractor-type $\times$ group ANOVA. This revealed a significant main effect of distractortype, $F(1,49)=30.86, p<.001, \eta_{\mathrm{p}}^{2}=.39$, with participants more likely to look at hightraining than low-training distractors, even though during this test phase the outcome 
signalled by each type of distractor had the same (low) value. The main effect of group was not significant, $F(1,49)=1.95, p=.17, \eta_{\mathrm{p}}^{2}=.04$. Importantly, the interaction between distractor-type and group was not significant, $F(1,49)=1.19, p=.28, \eta_{\mathrm{p}}^{2}=.024$.

To further analyse this non-significant interaction, we calculated distractor difference scores for each participant by taking the difference in the proportion of distraction trials between high- and low-training distractor trials; comparing these difference scores between Dev and DevFB groups via a Bayesian independent samples $t$-test yielded a Bayes factor of $B F_{01}=2.19$ in favour of the null hypothesis (no difference in attentional bias towards the high-training distractor between the two groups) over the alternative hypothesis. Notably, Figure 3A shows that the mean attentional bias was numerically (but not significantly: see analyses above) smaller in the Dev group than the DevFB group during the training phase, a pattern that carried through to the test phase. The implication is that any small difference between-group difference in attentional bias during the test phase was not a consequence of the difference in their treatment. Consistent with this idea, an analysis comparing the distractor difference scores in each group from the test phase, while controlling for the size of each group's attentional bias during the training phase (by using distractor difference scores from the training phase as a covariate in a Bayesian one-way ANOVA), yielded $B F_{01}=3.56$, suggesting substantial evidence in favour of the null hypothesis of no difference between the groups during the test phase (Jeffreys, 1961).

We conducted pre-planned analyses of simple effects to test the effect of distractor type in each group during the test phase. These analyses revealed a significant effect of distractor type in both the Dev group, $t(25)=3.14, p=.004, d=.62$, and the DevFB group, $t(24)=4.74, p<.001, d=.95$. In both groups participants were significantly more likely to look at the distractor that previously signalled a high-value fruit in the training phase relative to the distractor that previously signalled a low-value fruit. 
Knowledge checks. All participants correctly identified the current values of each fruit (the fruit-value contingencies) in the knowledge check following the test phase. In each group, only 2 participants failed to correctly identify which fruit was signalled by each coloured distractor (the colour-fruit contingencies). Re-analysis of proportion of distraction trials while excluding these participants who failed to correctly identify the colour-fruit contingencies revealed that the patterns of significant and non-significant findings were unchanged.

\section{Experiment $2 b$}

We first examined the proportion of distraction trials across the training and test phases of Experiment $2 \mathrm{~b}$ using a 2 (phase: training vs. test phase) $\times 2$ (distractor-type: highvs. low-training distractor) $\times 2$ (group: Super vs. SuperFB) ANOVA. Notably, the three-way interaction was not significant, $F(1,53)=0.006, p=.94, \eta_{\mathrm{p}}^{2}<.001$. Planned follow-up analyses focused on the proportion of distraction trials in the training and test phases separately

Training phase. Figure 4A shows proportion of distraction trials during the training phase, which were analysed using a distractor-type $\times$ group ANOVA. There was a significant main effect of distractor-type, $F(1,53)=15.18, p<.001, \eta_{\mathrm{p}}{ }^{2}=.22$, with more distraction trials when the display contained a high-training distractor than a low-training distractor. There was no significant main effect of group, $F(1,53)=0.04, p=.84, \eta_{\mathrm{p}}{ }^{2}<.001$, or distractor-type $\times$ group interaction, $F(1,53)=0.93, p=.34, \eta_{\mathrm{p}}{ }^{2}=.02$. 


\section{A) Training phase}

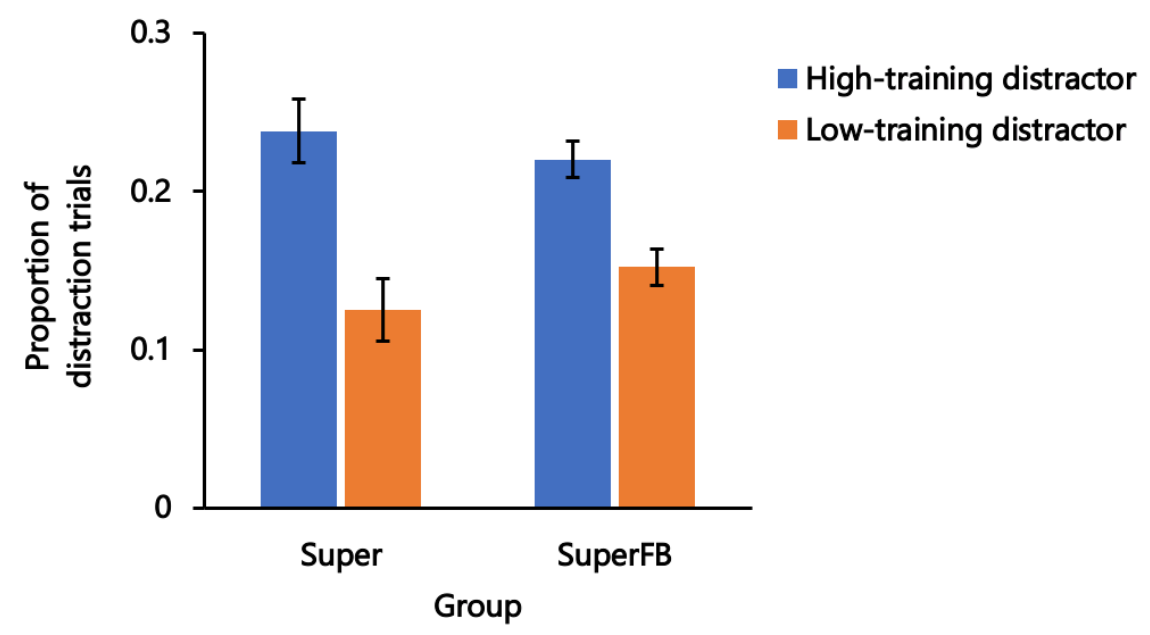

B) Test phase

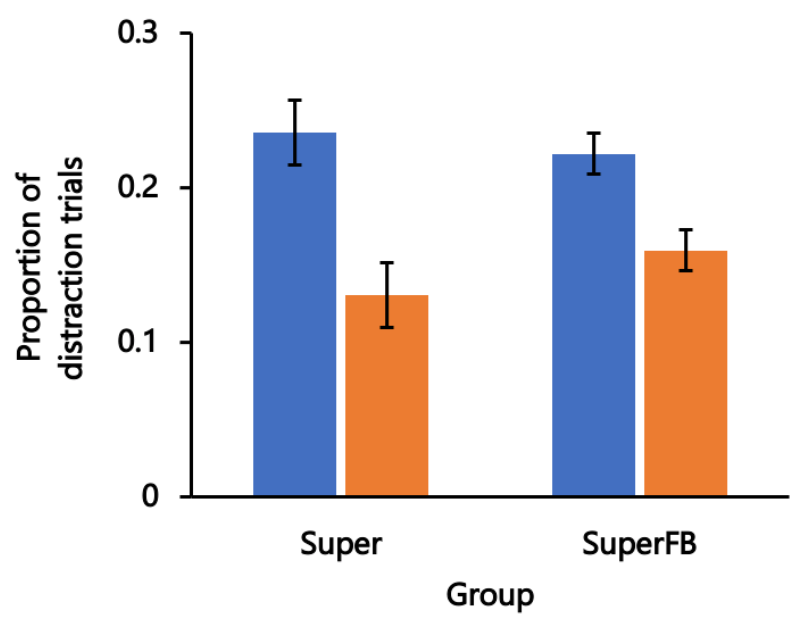

Figure 4. Proportion of distraction trials for trials featuring high- and low-training distractors in A) the training phase and B) the test phase of Experiment 2b. Distraction trials occurred when participants looked at the coloured distractor in the search display, resulting in omission of the fruit outcome on that trial. Note that distractors are defined by the value of the outcome they signalled during the training phase (e.g., a hightraining distractor was the colour associated with a high-value fruit outcome during training). Error bars show within-subjects SEM (Morey, 2008).

Test phase. Figure 4B shows the proportion of distraction trials for each group in the test phase, following the super-valuation of the low-value fruit. These data were again analysed using a distractor-type $\times$ group ANOVA. This revealed a significant main effect of distractor-type, $F(1,53)=11.33, p=.001, \eta_{\mathrm{p}}^{2}=.18$, participants more likely to look at hightraining than low-training distractors, even though during this test phase the outcome 
signalled by each type of distractor had the same (high) value. The main effect of group was not significant, $F(1,53)=0.12, p=.73, \eta_{\mathrm{p}}^{2}=.002$. Importantly, the interaction between group and distractor-type was not significant, $F(1,53)=0.74, p=.39, \eta_{\mathrm{p}}{ }^{2}=.014$.

To follow-up this non-significant interaction, we conducted a Bayesian independent samples $t$-test comparing distractor difference scores from the test phase. This analysis yielded a Bayes factor of $B F_{01}=2.71$ in favour of the null hypothesis (no difference in attentional bias towards the high-training distractor between the two groups) over the alternative hypothesis. When the size of each group's attentional bias during the training phase was further controlled for (by using distractor difference scores from the training phase as a covariate in a Bayesian one-way ANOVA), this analysis yielded $B F_{01}=3.67$, suggesting substantial evidence in favour of the null hypothesis of no difference between the groups during the test phase (Jeffreys, 1961).

We conducted pre-planned analyses of simple effects to test the effect of distractor type in each group during the test phase. These analyses revealed a significant effect of distractor type in both the Super group, $t(27)=2.52, p=.018, d=.45$, and the SuperFB group, $t(26)=2.37, p=.025, d=.46$. In both groups participants were significantly more likely to look at the distractor that previously signalled a high-value fruit in the training phase relative to the distractor that previously signalled a low-value fruit.

Knowledge checks. All participants correctly identified the current values of each fruit (the fruit-value contingencies) in the knowledge check following the test phase. Of participants who failed to correctly identify which fruit was signalled by each coloured distractor (the colour-fruit contingencies), five were in the Super group and three were in the SuperFB group. Re-analysis of proportion of distraction trials while excluding these participants who failed to correctly identify the colour-fruit contingencies revealed that the patterns of significant and non-significant findings were unchanged. 


\section{Discussion}

Findings from the training phases of Experiments $2 \mathrm{a}$ and $2 \mathrm{~b}$ replicated findings from Experiment 1: participants were more likely to look at the high-training distractor than the low-training distractor, even though this pattern meant that participants were more likely to miss out on high-value rewards.

In the test phase of Experiment 2a, participants in both groups continued to show a significant attentional bias towards the high-training distractor relative to the low-training distractor. This persistent prioritisation of the high-training distractor occurred even though it no longer signalled a high-value outcome, and in fact signalled a fruit outcome that was now of equal (low) value to the fruit associated with the low-training distractor. This pattern was also observed in the test phase of Experiment 2b; attentional prioritisation of the high-training distractor persisted even though the low-training distractor now signalled a fruit outcome of equal (high) value to that associated with the high-training distractor.

Strikingly, unlike Experiment 1 (where reversal of outcome values did seem to influence patterns of attention when feedback was provided), additional trial-by-trial feedback on the identity of the fruit earned on each trial (in groups DevFB and SuperFB) did not result in a change in the attentional prioritisation of the high-training distractor in line with its current value. Overall, then, devaluation of the previously-high-value outcome, and the super-valuation of a previously-low-value outcome, did not seem to have an effect on attention to a signal of that outcome. We postpone further interpretation of these findings to the General Discussion.

\section{General Discussion}

A defining feature of habitual behaviours is that they are not mediated or controlled by a representation of the outcome that results from that behaviour, and hence habitual responding is insensitive to acute changes in the value of that outcome (Dickinson \& 
Balleine, 1994). In the experiments presented here, we investigated whether conditioned attentional prioritisation of reward-related stimuli is sensitive to acute changes in the values of outcomes, and hence whether it can be considered a 'habit-like' response. In both experiments, participants were first trained to associate distractor stimuli with high- and lowvalue outcomes. Consistent with previous research (Le Pelley et al., 2015; Pearson et al., 2015, 2016), the extent to which participants had their attention captured by each distractor was modulated by the value of the outcome the distractor signalled. That is, participants were more often distracted by a stimulus that signalled a high-value outcome versus a low-value outcome. Looked at another way, the high-training distractor stimulus acquired a greater ability to elicit an attentional prioritisation response (than the low-training distractor stimulus) by virtue of its association with a high-value outcome. Notably, this pattern of results occurred even though looking at the high-training distractor was particularly counterproductive to participants' goals of maximising their payoff, as it resulted in the cancellation of a larger reward (relative to looking at the low-training distractor, which would have resulted in the cancellation of a smaller reward).

Interestingly, the relative attentional prioritisation of the high- and low-training distractors showed - under some conditions - evidence of sensitivity to changes in the values of outcomes. In Experiment 1, the values of outcomes were switched following the training phase in an instructed revaluation procedure: participants were told that the fruit which had previously had high value would now have low value, and vice versa. We found some evidence that this instructed switch in outcome values influenced participants' attentional prioritisation of distractors when tested in (nominal) extinction, such that the bias towards the high-training distractor was weaker than in a control group of participants for whom outcome values remained unchanged from training (with this difference reaching significance if analysis was restricted to participants who showed evidence of having learned colour-fruit 
associations in a final knowledge check). However, knowledge of the instructed revaluation alone was insufficient for participants to completely reverse their pattern of attentional bias so that it reflected the updated outcome values, implying that patterns of attentional prioritisation were only partially mediated by a representation of the fruit outcomes. By contrast, a 'full' reversal of attentional bias was observed only in a group of participants who received additional experience of receiving the outcome on each trial of the test phase in the form of feedback. This experience of explicit pairings of distractor colours and revalued outcomes in the test phase seemed to be sufficient for patterns of attentional prioritisation to reflect the changed values of the outcomes.

Under other conditions, previously learned reward-related attentional prioritisation responses showed clearer evidence of persistence in the face of changes in outcome values. In Experiment 2a, instructed devaluation following training rendered both fruits of equally low value; in Experiment 2b, instructed 'super-valuation' rendered both fruits of equally high value. Knowledge checks confirmed that participants knew and understood the updated values of the fruits, and yet in both experiments attentional prioritisation of the high-training distractor persisted. Notably, and unlike Experiment 1, this pattern of attentional prioritisation endured even when we provided participants with further experience of the distractoroutcome pairings in the form of feedback during the test phase.

Overall, the results of the current experiments specify conditions under which attentional prioritisation of reward-related stimuli may exhibit 'habit-like' persistence in the face of changing outcome values. Specifically, we observed evidence for persistence in Experiments $2 \mathrm{a}$ and $2 \mathrm{~b}$, when the values of both outcomes became equivalent in the test phase (whether that was via devaluation or super-valuation). At first glance this pattern suggests a habit-like value-independence of the reward-related attentional bias formed during training. Notably, however, similar persistence was also observed in participants who 
continued to receive outcome feedback during the test phase - that is, in participants who received further experience of distractor-outcome-value pairings, which should have led to updating of attentional priority even if this updating were based on a habit-like, experiencedependent process. By contrast, in Experiment 1, an instructed reversal of outcome values (with high- and low-value outcomes swapping) produced a weakening of the previously conditioned attentional bias; when further direct experience of distractor-outcome-value pairings was provided through continued feedback, this change in the pattern of attentional bias was greater, and indeed showed evidence of reversal to follow the updated outcome values.

We take this pattern of data to indicate the importance of motivation in the updating of attentional prioritisation responses. In Experiments $2 \mathrm{a}$ and $2 \mathrm{~b}$, since both outcomes were equally valuable during the test phase, participants may not have strived to earn one particular fruit over the other. Hence there may have been insufficient motivation for updating of attentional control settings to reflect the new fruit values, regardless of whether or not outcome feedback was provided, such that the previous pattern of reward-related attentional capture persisted across groups. In Experiment 1, the reversal of outcome values meant that there was still a difference in the relative value of trials in the test phase (see Kim $\&$ Beck, 2020, for recent work highlighting the importance of relative value in reward-related attentional bias). Consequently participants in the revaluation groups of Experiment 1 would have been more motivated to the now-high-value fruit than the now-low-value fruit - in turn motivating an update to attentional control settings in reflection of these changed values. The finding that this update was nevertheless incomplete in participants who did not receive feedback following outcome revaluation suggests in turn that outcome-value knowledge alone was insufficient to overcome the initially trained attentional bias: instead, further direct experience of pairings of stimuli with revalued outcomes was required. That is, the reward- 
related attentional bias formed during Phase 1 was only partially sensitive to changes in knowledge of the values of associated outcomes. The implication is that this attentional bias was - at least in part - independent of the current value of the outcome, and hence can be considered habit-like.

Our findings on the persistence of attentional prioritisation following outcome devaluation (Experiment 2a) are consistent with findings by De Tommaso et al. (2017, 2021). In the initial conditioning phase of their studies, thirsty participants learned that selecting a particular coloured cue had a high probability of earning a desired beverage outcome, whereas selecting other cues of other colours had lower probabilities of earning the outcome. This training produced an attentional bias towards the high-probability cue (as assessed in a subsequent visual-search test), and critically this bias persisted even after participants quenched their thirst, hence devaluing the beverage reward. That is, the high-reward stimulus maintained its attentional priority despite a reduction in the motivational value of the beverage outcome. However, there are some caveats that should be noted with regard to these prior studies. First, even after outcome devaluation in De Tommaso et al.'s procedure, the value of the high-probability cue (with a strong association to a weakly desired - but still appetitive - outcome) presumably remained higher than that of the low-probability cue (with a weak association to the same outcome). Consequently the high-probability cue remained the 'best' stimulus, and given the importance of relative value in determining reward-related attentional bias (Kim \& Beck, 2020) it is perhaps unsurprising that the attentional bias towards this high-probability cue persisted under these conditions. Second, in the visualsearch test phase of De Tommaso et al.'s procedure, the location of the search target was independent of the locations of reward-related colours; e.g., the target sometimes appeared in the same location as the high-probability cue, and sometimes in other locations, and hence there was no disadvantage in attending to reward-related cues in this task. As such, it is 
unclear whether the observed attentional bias reflects an automatic process of attentional capture (i.e., a change in the 'attention-grabbing' nature of the stimulus itself), or instead persistence of a more top-down prioritisation which continues to play out because it is not inconsistent with current goals. This contrasts with the current procedure, where attending to reward-signalling cues was explicitly counterproductive as it resulted in omission of rewards that would otherwise have been earned, so that attention to these cues was contrary to participants' goals. Finally, the requirement for participants to select the critical stimuli in the conditioning phase of De Tommaso et al.'s task may have led to the development of selection-based attentional biases rather than reward-based biases (Awh et al., 2012; Kyllingsbæk et al., 2001; Qu et al., 2017). By contrast, in the current procedure participants were never required to select the critical reward-signalling coloured stimuli, which only ever appeared as distractors.

Taking up this latter point, it is important to note that the relationship between the distractor stimuli and the delivery of reward in the current study is Pavlovian in nature distractors signalled the availability of reward, but participants were not required to respond to the distractors themselves in order to earn this reward. Typically, however, habits are considered in the behavioural neuroscience literature as involving instrumental relationships, where the organism learns that responding to the stimulus (e.g., a lever) is required for the delivery of the reward (e.g., a food pellet). This distinction is why we have referred to the attentional patterns observed in the current studies as 'habit-like'. In fact, the situation is complicated here because the response of attending to the coloured distractors does have an instrumental consequence in this task, albeit a negative one: omission of reward (see Pearson $\&$ Le Pelley, 2020, in press). Future research could examine this issue more closely, for example using a procedure in which attending to coloured stimuli is reinforced during an initial conditioning phase (followed by a test phase that is similar to the search task used in 
the current study) to investigate evidence for attentional habits in a more clearly instrumental preparation, which would potentially allow for closer and more direct links to be drawn with the literature on habits.

Another question that remains relates to the role of the amount of training on the development and persistence of 'habit-like' attentional prioritisation. One defining feature of a habit is that it is strengthened by stable and extensive training, with behaviour starting out as goal-directed (mediated by outcome value) and becoming habitual over the course of substantial experience (Adams, 1982; Dickinson, Balleine, Watt, Gonzalez, \& Boakes, 1995). The experiments presented here provided all participants with the same (moderate) amount of training, and hence do not allow us to probe the dynamics of a possible transition from outcome-mediated to outcome-independent attentional prioritisation. This idea could be studied in future research that investigates the effect of outcome revaluation on patterns of attentional prioritisation following varying amounts of training in Phase 1 of the attentional revaluation task.

\section{Attentional Habits and Behavioural Control}

The idea that attentional prioritisation can be a 'habit-like' response offers an alternative perspective on behavioural control. Behavioural choice and action represents the end-point of a chain of cognitive processes that eventuate in a final decision. This chain of information processing begins with attending to, and encoding, the stimuli present in the environment, and consequently attention has the potential to fundamentally shape our behaviour: if we do not notice that a stimulus is present, it is unlikely that that stimulus will be a target of our overt behaviour; conversely if our attentional system flags a particular stimulus as being significant, then it is more likely to be a focus of subsequent action. The importance of attention in decision-making becomes more apparent when considering realworld scenarios with numerous behavioural options; e.g., when shopping at the supermarket, 
we are confronted with thousands of potential targets of overt choice. In such a situation, we cannot carefully weigh up the pros and cons of every possible option before making a choice. Instead attention will play a critical role here in prioritising some options for further consideration, and filtering out others so that they place no further demand on cognitive resources. This process of prioritisation will be guided in part by our current goals - if we have run out of milk we will prioritise the dairy section - but demonstrations of rewardrelated attentional capture suggest that this filtering will also be influenced by our previous experiences of outcome value. Items that have previously been associated with reward (e.g., tasty chocolate) may be more likely to "jump out" at us from the display, and hence we may be more likely to buy them (see Gluth et al., 2018, 2020). Notably, the current demonstration that reward-related attention can be habit-like suggests that this automatic prioritisation may persist even when the values of items change, for example if we decide to go on a diet so that chocolate is no longer desired. In effect, this account proposes that attention acts to automatically and habitually prioritise certain possible goals and related courses of action (cf. Cushman \& Morris, 2015). Taken further, this process may play a role in maintaining maladaptive behaviours towards reward-related stimuli implicated in addiction and compulsive disorders despite attempts to abstain. Consistent with this idea, reward-related attentional capture has been shown to be associated with illicit drug use (Albertella et al., 2017), risky alcohol use (Albertella, Watson et al., 2019), and trait compulsivity and impulsivity (Albertella, Le Pelley et al., 2019), and to predict the likelihood of temporary abstinence success (Albertella et al., 2020). 


\section{Conclusions}

In line with previous research, the current study demonstrated that reward learning exerts an influence on the allocation of attentional priority; stimuli associated with large rewards were more likely to elicit an attentional prioritisation response than stimuli associated with small rewards, even though attending to these stimuli was contrary to participants' goals (Le Pelley et al., 2015; Pearson et al., 2015, 2016). Crucially, our findings highlighted that under some conditions, this counterproductive attentional prioritisation can be considered 'habit-like', becoming divorced from a representation of the associated outcome, and hence insensitive to acute changes in the value of that outcome. Namely, the attentional prioritisation of a stimulus signalling a (previously) high-value outcome persisted following instructed devaluation and super-valuation. Interestingly, following a value-switch manipulation (wherein motivation remained high and there was a relative difference in the values of outcomes), there was evidence of partial sensitivity of attentional prioritisation to acute changes in the values of outcomes. Under these conditions, it appears that knowledge of the instructed value-switch alone was insufficient for participants to completely reverse their pattern of attentional bias so that it reflected the updated outcome values; a 'full' reversal of the attentional bias required additional experience of the stimulus-outcome pairings. Taken together, these findings provide a framework for future work investigating the relationship between attention and behavioural control. 


\section{References}

Adams, C.D. (1982). Variations in the sensitivity of instrumental responding to reinforcer devaluation. Quarterly Journal of Experimental Psychology, 34B, 77-98.

Adams, C.D., \& Dickinson, A. (1981). Instrumental responding following reinforcer devaluation. Quarterly Journal of Experimental Psychology, 33B, 109-122.

Albertella, L., Copeland, J., Pearson, D., Watson, P., Wiers, R. W., \& Le Pelley, M. E. (2017). Selective attention moderates the relationship between attentional capture by signals of nondrug reward and illicit drug use. Drug and Alcohol Dependence, 175, 99-105.

Albertella, L., Le Pelley, M. E., Chamberlain, S. R., Westbrook, R. F., Fontenelle, L., Segrave, R., Lee, R., Pearson, D. \& Yücel, M. (2019). Reward-related attentional capture is associated with severity of addictive and obsessive-compulsive behaviors. Psychology of Addictive Behaviors, 33, 495-502.

Albertella, L., Vd Hooven, J., Bovens, R., \& Wiers, R. W. (2021). Reward-related attentional capture predicts non-abstinence during a one-month abstinence challenge. Addictive Behaviors, 114, 106745.

Albertella, L., Watson, P., Yücel, M., \& Le Pelley, M.E. (2019). Persistence of valuemodulated attentional capture is associated with risky alcohol use. Addictive Behaviors Reports, 10, 100195

Anderson, B. A. (2016). The attention habit: How reward learning shapes attentional selection. Annals of the New York Academy of Sciences, 1369(1), 24-39.

Anderson, B. A., Folk, C. L., Garrison, R., \& Rogers, L. (2016). Mechanisms of habitual approach: Failure to suppress irrelevant responses evoked by previously rewardassociated stimuli. Journal of Experimental Psychology: General, 145(6), 796-805. 
Anderson, B. A., \& Halpern, M. (2017). On the value-dependence of value-driven attentional capture. Attention, Perception, \& Psychophysics, 79(4), 1001-1011.

Awh, E., Belopolsky, A. V., \& Theeuwes, J. (2012). Top-down versus bottom-up attentional control: A failed theoretical dichotomy. Trends in Cognitive Sciences, 16(8), 437-443.

Berridge, K. C., \& Robinson, T. E. (1998). What is the role of dopamine in reward: hedonic impact, reward learning, or incentive salience?. Brain Research Reviews, 28(3), 309369.

Brainard, D.H. (1997). The psychophysics toolbox. Spatial Vision, 10, 433-436.

Cushman, F., \& Morris, A. (2015). Habitual control of goal selection in humans. Proceedings of the National Academy of Sciences of the United States of America, 112, 1381713822.

De Tommaso, M., Mastropasqua, T., \& Turatto, M. (2017). The salience of a reward cue can outlast reward devaluation. Behavioral Neuroscience, 131(3), 226-234.

De Tommaso, M., \& Turatto, M. (2021). On the resilience of reward cues attentional salience to reward devaluation, time, incentive learning, and contingency remapping. Behavioral Neuroscience, 135(3), 389-401.

Deubel, H., \& Schneider, W. X. (1996). Saccade target selection and object recognition:

Evidence for a common attentional mechanism. Vision Research, 36(12), 1827-1837.

Dickinson, A. (1985). Actions and habits: The development of behavioural autonomy. Philosophical Transactions of the Royal Society of London. B, Biological Sciences, 308(1135), 67-78.

Dickinson, A., \& Balleine, B. (1994). Motivational control of goal-directed action. Animal Learning \& Behavior, 22(1), 1-18. 
Dickinson, A., Balleine, B., Watt, A., Gonzalez, F., \& Boakes, R. A. (1995). Motivational control after extended instrumental training. Animal Learning \& Behavior, 23(2), 197-206.

Failing, M., Nissens, T., Pearson, D., Le Pelley, M., \& Theeuwes, J. (2015). Oculomotor capture by stimuli that signal the availability of reward. Journal of Neurophysiology, 114, 2316-2327.

Failing, M., \& Theeuwes, J. (2018). Selection history: How reward modulates selectivity of visual attention. Psychonomic Bulletin \& Review, 25, 514-538.

Gluth, S., Kern, N., Kortmann, M., \& Vitali, C. L. (2020). Value-based attention but not divisive normalization influences decisions with multiple alternatives. Nature Human Behaviour, 4, 634-645.

Gluth, S., Spektor, M. S., \& Rieskamp, J. (2018). Value-based attentional capture affects multi-alternative decision making. ELife, 7, e39659.

Hickey, C., Chelazzi, L., \& Theeuwes, J. (2010). Reward changes salience in human vision via the anterior cingulate. Journal of Neuroscience, 30(33), 11096-11103.

JASP Team. (2020). JASP (Version 0.13.1) [Computer software]. https://jasp-stats.org/

Jeffreys, H. 1961. Theory of probability (3rd ed.). New York: Oxford University Press.

Jiang, Y. V., \& Sisk, C. A. (2019). Habit-like attention. Current Opinion in Psychology, 29, $65-70$.

Kim, S., \& Beck, M. R. (2020). Impact of relative and absolute values on selective attention. Psychonomic Bulletin \& Review, 27(4), 735-741.

Kleiner, M., Brainard, D.H., \& Pelli, D.G. (2007). What's new in Psychtoolbox-3? Perception, 36, ECVP Abstract Supplement.

Kyllingsbæk, S., Schneider, W.X., \& Bundesen, C. (2001). Automatic attraction of attention to former targets in visual displays of letters. Perception \& Psychophysics, 63, 85-98. 
Le Pelley, M. E., Mitchell, C. J., Beesley, T., George, D. N., \& Wills, A. J. (2016). Attention and associative learning in humans: An integrative review. Psychological Bulletin, 142(10), 1111-1140.

Le Pelley, M. E., Pearson, D., Griffiths, O., \& Beesley, T. (2015). When goals conflict with values: Counterproductive attentional and oculomotor capture by reward-related stimuli. Journal of Experimental Psychology: General, 144(1), 158-171.

Le Pelley, M.E., Watson, P., Pearson, D., Abeywickrama, R.S., \& Most, S.B. (2019). Winners and losers: Reward and punishment produce biases in temporal selection. Journal of Experimental Psychology: Learning, Memory, \& Cognition, 45, 822-833.

Luque D., Beesley T., Morris R. W., Jack B. N., Griffiths O., Whitford T. J. \& Le Pelley M. E. (2017). Goal-directed and habit-like modulations of stimulus processing during reinforcement learning. Journal of Neuroscience, 37, 3009-3017.

Luque D., Molinero S., Watson P., López F. J. \& Le Pelley M. E. (2020). Measuring habit formation through goal-directed response switching. Journal of Experimental Psychology: General, 149, 1449-1459.

Morey, R.D. (2008). Confidence intervals from normalized data: A correction to Cousineau (2005). Tutorial in Quantitative Methods for Psychology, 4, 61-64.

Pearson, D., Donkin, C., Tran, S. C., Most, S. B., \& Le Pelley, M. E. (2015). Cognitive control and counterproductive oculomotor capture by reward-related stimuli. Visual Cognition, 23(1), 41-66.

Pearson, D., \& Le Pelley, M.E. (2020). Learning to avoid looking: Competing influences of reward on overt attentional selection. Psychonomic Bulletin \& Review, 27, 998-1005.

Pearson D. \& Le Pelley M. E. (in press). Reward encourages reactive, goal-directed suppression of attention. Journal of Experimental Psychology: Human Perception and Performance. 
Pearson, D., Osborn, R., Whitford, T. J., Failing, M., Theeuwes, J., \& Le Pelley, M. E. (2016). Value-modulated oculomotor capture by task-irrelevant stimuli is a consequence of early competition on the saccade map. Attention, Perception, \& Psychophysics, 78(7), 2226-2240.

Qi, S.Q., Zeng, Q.H., Ding, C., \& Li, H. (2013). Neural correlates of reward-driven attentional capture in visual search. Brain Research, 1532, 32-43.

Qu, Z., Hillyard, S.A., \& Ding, Y.L. (2017). Perceptual learning induces persistent attentional capture by non-salient shapes. Cerebral Cortex, 27, 1512-1523.

Raymond, J. E., \& O’Brien, J. L. (2009). Selective visual attention and motivation: The consequences of value learning in an attentional blink task. Psychological Science, 20, 981-988.

Rusz, D., Le Pelley, M.E., Kompier, M.A.J., Mait, L., \& Bijleveld, E. (2020). Reward-driven distraction: A meta-analysis. Psychological Bulletin, 146, 872-899.

Tricomi, E., Balleine, B. W., \& O’Doherty, J. P. (2009). A specific role for posterior dorsolateral striatum in human habit learning. European Journal of Neuroscience, 29(11), 2225-2232.

Watson, P., Pearson, D., Chow, M., Theeuwes, J., Wiers, R. W., Most, S. B., \& Le Pelley, M. E. (2019a). Capture and control: Working memory modulates attentional capture by reward-related stimuli. Psychological Science, 30(8), 1174-1185.

Watson, P., Pearson, D., \& Le Pelley, M. E. (2020). Reduced attentional capture by reward following an acute dose of alcohol. Psychopharmacology, 237(12), 3625-3639

Watson, P., Pearson, D., Most, S.B., Theeuwes, J., Wiers, R.W., \& Le Pelley, M.E. (2019b). Attentional capture by Pavlovian reward-signalling distractors in visual search persists when rewards are removed. PLoS ONE, 14, e0226284. 
Watson, P., van Wingen, G. \& de Wit, S. (2018). Conflicted between goal-directed and habitual control, an fMRI investigation. Eneuro, 5(4), e0240-18.2018.

Yantis, S. (2000). Goal-directed and stimulus-driven determinants of attentional control. In S. Monsell \& J. Driver (Eds.), Attention and performance (pp. 73-103). Cambridge, MA: MIT Press. 